\title{
AGN Activity in Brigtest Cluster Galaxies (BCGs)
}

\author{
Süleyman Fişek ${ }^{* 1,2}$, Sinan Alişs,2, E. Kaan Ülgen ${ }^{1}$, and F. Korhan Yelkenci ${ }^{1}$ \\ ${ }^{1}$ Istanbul University, Science Faculty, Department of Astronomy and Space Sciences, Istanbul, Turkey \\ ${ }^{2}$ Istanbul University Observatory Application and Research Center, Istanbul, Turkey
}

\begin{abstract}
The aim of our study is to classify the Brightest Cluster Galaxies (BCG), which are the brightest and most massive galaxies in the universe and to determine fraction of BCGs showing AGN activity. Within the scope of the study, we combine BCGs from galaxy cluster catalogs which presented by Hao et al. (2010) and Wen et al. (2009) constructed from the SDSS (Sloan Digital Sky Survey) data and BCGs identified from CFHTLS (Canada-France-Hawaii Telescope Legacy Survey) Deep fields (Alis, 2009). Our combined sample contains 42490 BCGs. We determine activity types of BCGs by means of BPT diagrams and WISE color-color diagrams. 140 BCGs show AGN activity out of 42490. In addition, we calculated star formation rates (SFR) by using $\mathrm{H} \alpha$ emission lines for 5569 BCGs with suitable spectral data and investigated the correlation between SFR and redshift.
\end{abstract}

Keywords: Galaxy: AGN - Galaxy: BCG - Galaxy: evolution - Galaxy: star formation

\section{Introduction}

BCGs are the brightest and the most massive galaxies in the universe and usually located in the center or near the center of rich galaxy clusters (Jones \& Forman, 1984, Smith et al., 2005). Most of the BCGs are red elliptical galaxies and star formation activities are almost stopped (Edwards et al., 2007). The evolutionary processes of BCGs is still not fully understood; galaxy-galaxy interactions and galaxy mergers are thought to be effective for the process of formation of these galaxies where there is intense interactions (Edwards et al., 2007).

As a result of these interactions within the cluster, an increase in the star formation activity of the BCGs can be observed, unlike the other elliptical galaxies in the cluster (Liu et al., 2012, O'Dea et al., 2008, Pipino et al., 2009). Investigating star formation activity in BCGs can help us to understand the evolution of the cluster and environment as a function of redshift.

\section{Data}

BCGs examined in this study collected from two different datasets. 89 BCGs were determined by Aliş et al. (Alis, 2009, Aliş et al., 2012) constitute our first dataset. These galaxies identified using a set of 159 galaxy cluster identified by Olsen et al. (2007) using five bands (u, g, r, i, z) from the CFHTLS-Deep Survey.

The second dataset used in our study are constructed from Hao et al. (2010) and Wen et al. (2009), where galaxy clusters identified from the SDSS. In the list given by Wen et al. (2009), 39716 galaxy clusters in the range of $0.05<\mathrm{z}<0.6$ were determined using SDSS DR6 data. Hao et al. (2010) listed 55427 galaxy clusters in the range of $0.1<\mathrm{z}<0.55$ using DR7 data. When the lists are combined, with overlapping galaxy clusters taken into account, the total number of galaxy clusters become 82164 . Merged cluster catalog of CFHTLS and SDSS contains 82253 clusters hence BCGs.

${ }^{*}$ sfisek@istanbul.edu.tr 
In this study, emission line fluxes of $\mathrm{H} \alpha$ [6562], $\mathrm{H} \beta$ [4861], OIII[5006], OI[6300], NII[6583], SII[6716] and spectroscopic redshifts of BCGs were obtained from the SDSS DR12 (i.e. emissionLinesPort) (http://www.sdss.org/dr12/). These emission line fluxes are used to determine the activity type of BCGs and also to calculate the star formation rates.

For the 42490 BCGs out of 82253 we could obtain spectroscopic data from SDSS. First we keep fluxes with $(\mathrm{F}(H \alpha)>0)$ for SFR calculation. In order to use the most reliable flux measurements, we eliminated the flux values with associated errors very high. For this purpose, we only used flux measurements with a relative error less than $50 \%$ for $H \alpha$. Besides flux error we also used signal to noise ratio $(\mathrm{S} / \mathrm{N})$ for keeping high quality fluxes. In a similar study Liu et al. (2012) used $\mathrm{S} / \mathrm{N}>3$. Therefore, we created two subsamples one is with $\mathrm{S} / \mathrm{N}>3$ and the other one is with $\mathrm{S} / \mathrm{N}>15$. In both samples we kept the fluxes with relative errors less than $50 \%$.

Among 42490 BCGs with relevant flux measurements, there are 1137 galaxies satisfying the flux error and $\mathrm{S} / \mathrm{N}$ criteria and only 143 of them can be used in the BPT diagram. When we loose the $\mathrm{S} / \mathrm{N}$ criteria as $\mathrm{S} / \mathrm{N}>3,5613$ galaxies with appropriate fluxes for SFR calculation and 410 galaxies suitable for BPT diagram remain.

The infrared photometric data used in this study were obtained from the AllWISE catalog, which is the all sky survey by WISE (Wide-field Infrared Survey Explorer) satellite. We obtained W1, W2, W3 and W4 infrared magnitudes for our galaxies from NASA/IPAC Infrared Science Archive (http://irsa.ipac.caltech.edu/Missions/wise.html).

\section{Method}

\subsection{Determining Activity Types of Galaxies}

In this study, we use two different methods to determine the activity types of BCGs.

\section{BPT Diagram:}

The first method is the BPT diagram (Baldwin et al., 1981). In this diagram, it is possible to classify galaxies according to their activity by using $\mathrm{H} \alpha$ [6562], $\mathrm{H} \beta$ [4861], OIII[5007], OI[6300], NII[6583], SII[6717] emission line fluxes. In addition, the BPT diagram is a tool used in the literature to determine whether galaxies show star formation activity.

\section{WISE Color - Color Diagram:}

From the photometric data obtained by the WISE satellite, the W1 $(3.4 \mu)$ and W2 $(4.6 \mu)$ bands are sensitive to the stellar mass of galaxies and the W3 $(12 \mu)$ band is sensitive to the star formation rates (Cluver et al., 2014, Fraser-McKelvie et al., 2014).

Cluver et al. (2014) matched the GAMA http://www.gama-survey.org Survey and WISE data to examine the infrared properties of more than 110,000 galaxies. Infrared color - color diagrams $((W 2-W 3)-(W 1-W 2))$ were created where galaxies with $W 2-W 3>1.5$ show mostly star formation activity.

\subsection{Star Formation Rate (SFR) Calculation}

The $\mathrm{H} \alpha$ emission flux is one of the most reliable and widely used tool to determine star formation in galaxies. The $\mathrm{H} \alpha$ line, seen as an emission in a galaxy spectrum, is an indicator of the star formation activity in galaxies.

In this study, we calculated star formation rates of the galaxies using (1) equation given by Kennicutt (1998).

$$
\operatorname{SFR}\left(M_{\odot} / \text { year }\right)=7.9 \times 10^{-42} \times L(H \alpha)\left(\text { ergs }^{-1}\right)
$$


As a first step, emission line fluxes obtained from SDSS needed to be converted to $\mathrm{H} \alpha$ luminosity. Thus, we computed the distances of our BCGs using their spectroscopic redshifts. Within the $\Lambda$ CDM cosmology, we determined the luminosity distances with $\Omega_{m}=0.3, \Omega_{\Lambda}=0.7$ ve $H_{0}=70 \mathrm{~km} / \mathrm{s} / \mathrm{Mpc}$ (Hogg, 1999). The distance calculations were made using the Python version of Ned Wright's cosmology calculator (http://www.astro.ucla.edu/ wright/CosmoCalc.html) (Wright, 2006). This Python program used by adapting it into the SFR script that we developed (Fişek, 2018).

Once the distances are known it is straightforward to obtain $\mathrm{H} \alpha$ luminosities by the equation (2):

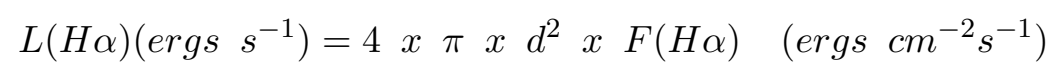

After calculating the $\mathrm{H} \alpha$ luminosity, the star formation rates were calculated for each galaxy by using equation (1).

\section{Results}

\subsection{BCGs by Type}

\section{Using by BPT Diagram:}

In this study, We show the BPT diagram produced from the spectra with $\mathrm{S} / \mathrm{N}>15$ is in Figure 1 for the galaxies we have examined in this study.
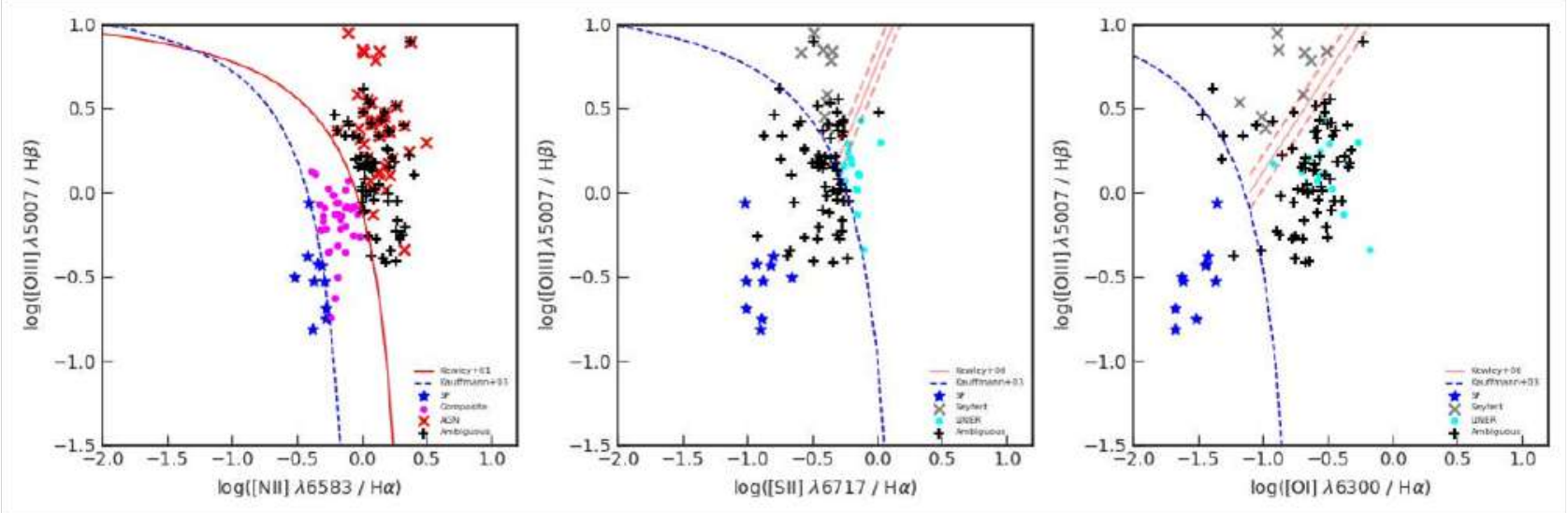

Figure 1. BPT diagram for galaxies with flux error less than $50 \%$ and $\mathrm{S} / \mathrm{N}>15$. The BPT diagrams show star forming galaxies (blue stars - SF), composite galaxies (pink dots - Comp), AGNs (red crosses), Seyfert Galaxies (gray crosses), LINER Galaxies (turquoise crosses), and "ambiguous" galaxies (black pluses).

\section{Using by WISE Diagram:}

Galaxies classified by the BPT diagram are shown in the WISE color - color diagram in Figure 2. 18 galaxies with $\mathrm{S} / \mathrm{N}>15$ in the BPT diagram couldn't be used in the WISE color - color diagram since the WISE magnitudes couldn't be measured for these galaxies. In addition, all the galaxies identified as SF in the BPT diagram are also determined as SF according to the WISE color - color diagrams.

\subsection{Star Formation Rates of BCGs}

SFR of $5569(\mathrm{~S} / \mathrm{N}>3)$ and $1136(\mathrm{~S} / \mathrm{N}>15)$ galaxies were determined and the distribution of these SFRs are shown in Figure 3 a.

\section{Correlation between SFR and Redshift:}




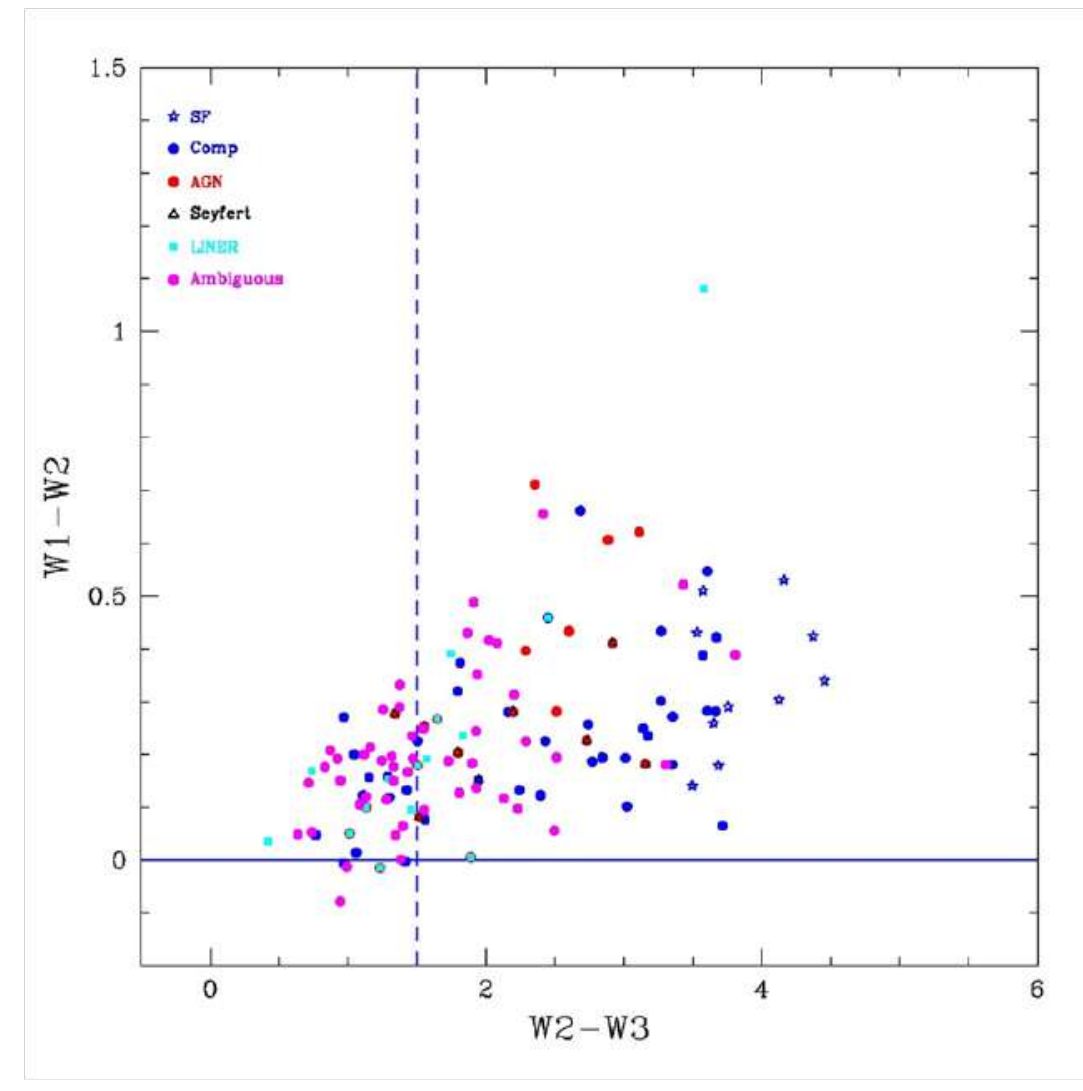

Figure 2. WISE color - color diagram for galaxies with flux error less than $50 \%$ and $\mathrm{S} / \mathrm{N}>15$. The dashed line indicates $W 2-W 3=1.5$. In the graph, galaxies are marked according to the BPT classes.

In order to check whether SFRs of BCGs evolve with redshift, we computed the mean SFRs with redshift bins of 0.1 . When there are less galaxies than 10 in any bin, corresponding statistical values are not used. Figure $3 \mathrm{~b}$ shows the SFR as a function of redshift.

\section{Discussion and Conclusion}

In this study, we investigated star formation activity of BCGs identified from CFHTLS and SDSS.

There is no clear correlation between the SFR of BCGs and their redshift. Similar result was presented by Cooke et al. (2014) in their study of 42 BCGs in the same redshift range as in this study. However, as shown in Figure $3 \mathrm{~b}$, it can be seen that the mean SFRs obtained after discarding outliers (i.e. $3 \sigma$ ) decrease towards lower redshifts. This is consistent star formation history of the universe as given by Madau \& Dickinson (2014). However, BCGs can be expected to behave differently from this general trend due to their environment. Environment plays an important role especially in the galaxy clusters with cooling cores. There have been studies where BCGs in cooling core clusters show induced star formation activity (Pipino et al., 2009, Voit, 2005). However, this behaviour is not well understood and needs to be investigated further.

Largest spectroscopic data available so far is the SDSS but with SDSS it is not possible to reach redshifts beyond $\mathrm{z} \sim 0.5$. Thus, wide area spectroscopic surveys with larger telescopes are needed in order to understand evolution of these galaxies. It seems this will become possible with the Mauna Kea Spectroscopic Explorer (MSE) in the near future. 

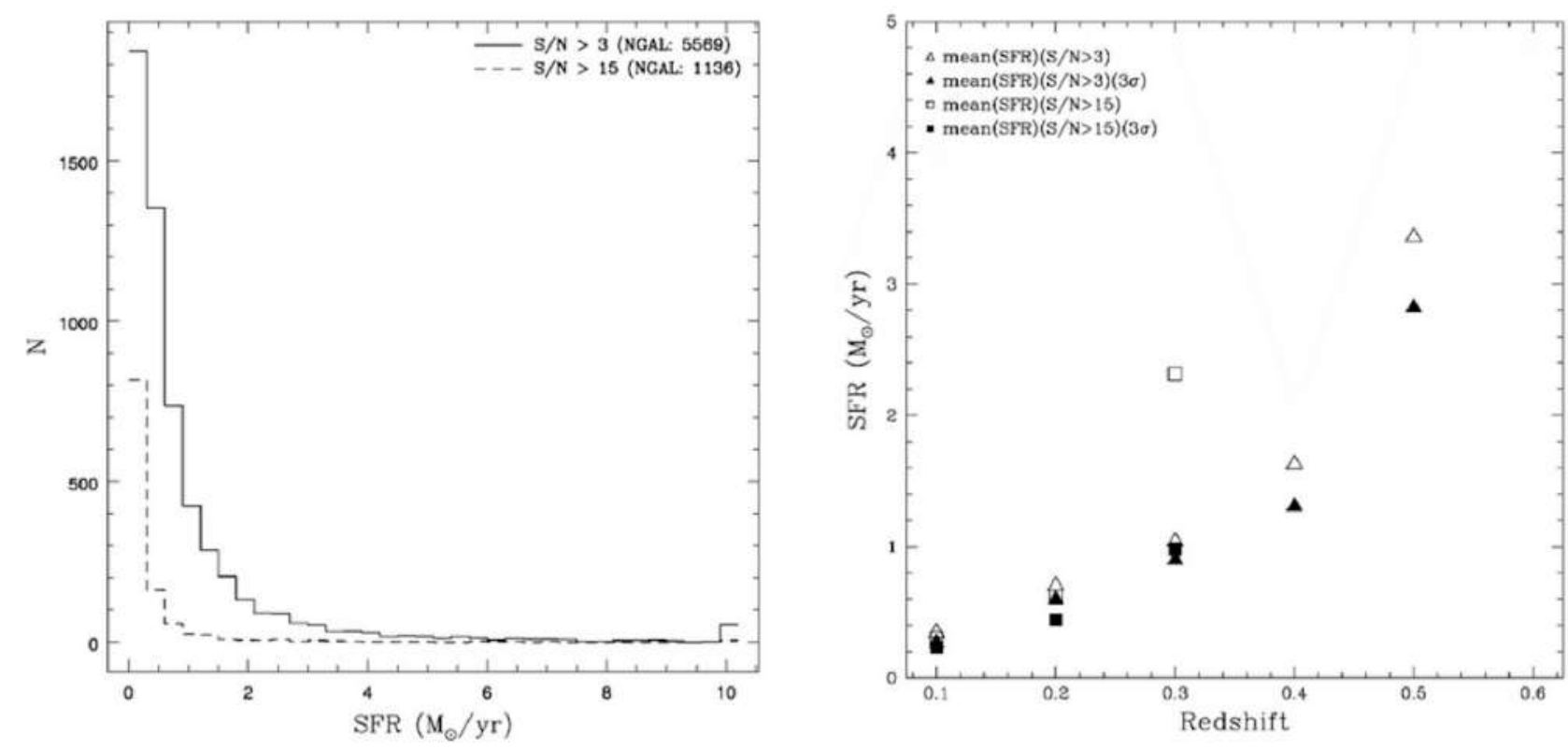

Figure 3. (a)Distribution of calculated SFR values (for SFR $<10 M_{\odot} /$ year). The solid line indicates galaxies with $\mathrm{S} / \mathrm{N}>3$ and the dashed line indicates galaxies with $\mathrm{S} / \mathrm{N}>15$. (b) Variation of the mean SFR of the galaxies with redshift for the galaxies with flux error less than $50 \%$ and $\mathrm{S} / \mathrm{N}>3$ or $\mathrm{S} / \mathrm{N}>15$. Empty triangles represent the mean SFR for galaxies with $\mathrm{S} / \mathrm{N}>3$ and empty squares are for galaxies with $\mathrm{S} / \mathrm{N}>15$. The filled symbols show values when outliers (i.e. $3 \sigma$ ) are not taken into account. 


\section{References}

\section{References}

Alis S., 2009, Publications de l'Observatoire Astronomique de Beograd, 86, 309

Aliş S., Yelkenci K., Benoist C., Limboz F., 2012, XVIII. National Astronomy Congress of TURKEY Proceedings Book, p. 345

Baldwin J. A., Phillips M. M., Terlevich R., 1981, Publications of the Astronomical Society of the Pacific, 93, 5

Cluver M. E., Jarrett T. H., Hopkins A. M., et al. 2014, The Astrophysical Journal, 782, 17

Cooke K. C., O’Dea C. P., Baum S. A., et al. 2014, The Astrophysical Journal, 833, 10

Edwards L. O. V., Hudson M. J., Balogh M. L., Smith R. J., 2007, The Astrophysical Journal, 379, 100

Fişek S., 2018, Istanbul University, Graduate School of Engineering and Science, p. 1

Fraser-McKelvie A., Brown M. J. I., Pimbblet K. A., 2014, Monthly Notices of the Royal Astronomical Society, 444, 63

Hao J., McKay T. A., Koester B. P., et al. 2010, The Astrophysical Journal Supplement Series, 191, 254

Hogg D. W., 1999, arXiv:astro-ph, 5116

Jones C., Forman W., 1984, The Astrophysical Journal, 276, 38

Kennicutt R. C. J., 1998, Annual Review of Astronomy and Astrophysics, 36, 189

Liu F. S., Mao S., Meng X. M., 2012, Monthly Notices of the Royal Astronomical Society, 423, 422

Madau P., Dickinson M., 2014, Annual Review of Astronomy and Astrophysics, 52, 415

O’Dea C. P., Baum S. A., Privon G., et al. 2008, The Astrophysical Journal, 681, 1035

Olsen L. F., Benoist C., Cappi A., et al. 2007, Astronomy \& Astrophysics, 461, 81

Pipino A., Kaviraj S., Bildfell C., et al. 2009, Monthly Notices of the Royal Astronomical Society, 395, 462

Smith G. P., Kneib J. P., Smail I., et al. 2005, Monthly Notices of the Royal Astronomical Society, 359,417

Voit G. M., 2005, Reviews of Modern Physics, 77, 207

Wen Z. L., Han J. L., Liu F. S., 2009, The Astrophysical Journal Supplement Series, 183, 197

Wright E. L., 2006, The Publications of the Astronomical Society of the Pacific, 118, 1711 